\title{
Adherencia al tratamiento antihipertensivo: ¿por qué aún no despega, y en qué medida influye en el control de la Presión Arterial?
}

Tomás Romero

School of Medicine, Department of Cardiology, University of California, San Diego. Institutional Review Board, Sharp

Health Care, San Diego, California

"El Señor ha creado medicinas en el mundo y quien es sabio no las rechazará". Ecclesiasticus 38:4.

"Es fácil obtener mil prescripciones, pero difícil lograr una simple mejoría". Proverbio Chino.

La hipertensión arterial es el mayor factor de riesgo de morbilidad y mortalidad de las enfermedades cardiovasculares en Chile y en el resto del mundo y por lo tanto aparece como el factor de riesgo mas relevante en el conjunto de enfermedades crónicas no transmisibles ${ }^{1-2-3-4-5}$. Sin embargo, el control insatisfactorio de la presión arterial (PA) de acuerdo a lo recomendado por las guías clínicas de uso actual ( $<140 / 90 \mathrm{mmHg}$ ) es un problema persistente en la población hipertensa en todas las regiones del mun$\mathrm{do}^{2-3-4-5}$. Un factor determinante es la falta de acceso al tratamiento médico, que fluctúa desde el $90 \%$ en las regiones más pobres hasta menos del $20 \%$ en los países de mayor ingreso ${ }^{2-4}$. Pero de aquellos hipertensos que reciben tratamiento no más del $50 \%$ logran controlar su $\mathrm{PA}^{2-3-4}$ 5-6-7. Se estima que para el año 2025, si estas tendencias no mejoran, habrán alrededor de 750 millones de hiper- tensos no controlados en el mundo ${ }^{8}$. En Chile, de acuerdo a la Encuesta Nacional de Salud (ENS) del año 2010, la prevalencia de hipertensión era 26.9\%, 37.26\% recibían tratamiento pero solo $16.9 \%$ de la población general de hipertensos tenían una PA controlada $(<140 / 90 \mathrm{~mm} / \mathrm{Hg})^{5}$ Factores determinantes de esta brecha entre el acceso al tratamiento de los hipertensos y el logro de resultados satisfactorios son:

1) Falta de adherencia al tratamiento prescrito. ${ }^{2-3-6-7-10}$

2) Persistencia de estilos de vida y hábitos desfavorables (dieta rica en sodio y en calorías, pobre en frutas y verduras, sedentarismo, obesidad). ${ }^{2-3-11}$

3) Tratamientos ineficientes ("inercia terapéutica" o falta de ajustes necesarios de dosificación y/o uso de fárma$\cos ) .^{12}$

En esta breve revisión nos limitaremos a discutir algunos de los factores relacionados con la adherencia terapéutica y en especial al tratamiento farmacológico antihipertensivo y su impacto en el control de la PA.

\section{Correspondencia:}

Dr. Tomás Romero

School of Medicine, Department of Cardiology, University of California, San Diego. Institutional

Review Board, Sharp Health Care, San Diego, California

tromero560@aol.com 
I. Aspectos generales de la adherencia a la farmacoterapia. De acuerdo a un informe de la Organización Mundial de la Salud la adherencia a la farmacoterapia en general se ha mantenido sin variaciones en los últimos 30 años, oscilando entre el 20-50\%, mayor en las enfermedades agudas y menor en las crónicas. Si no ocurren cambios favorables, la proporción de no adherentes a la farmacoterapia irá en aumento con el envejecimiento progresivo de la población y la consiguiente mayor prevalencia de enfermedades crónicas ${ }^{9-13}$.

\section{Factores generalmente relacionados con la baja ad- herencia.}

1) Socio-económicos: bajo nivel educacional e ingreso del paciente, costo de los medicamentos. ${ }^{2-4-9-10-14-15}$

2) Sistemas de Salud y proveedores: acceso limitado, horarios inapropiados, esperas prolongadas, relación médico paciente insatisfactoria. ${ }^{9-10-15}$

3) Dependientes de los pacientes, de sus enfermedades y/o tratamientos: factores emocionales y cognitivos, polifarmacia (múltiples medicamentos y dosificación), falta de síntomas (frecuente en hipertensos y diabéticos), efectos secundarios de los medicamentos, enfermedades crónicas, alcoholismo. ${ }^{9-10-5-16}$

\section{Prevalencia de la Adherencia a la farmacoterapia antihipertensiva:}

Existe bastante disparidad en las cifras publicadas en la literatura, dependiendo del tipo de estudios y de los métodos utilizados para medirla. En los ensayos clínicos en que los pacientes son enrolados en forma voluntaria y a quienes habitualmente se les proveen sin costo los medicamentos y son sujetos a un seguimiento riguroso la adherencia suele ser entre el 50-80\%, en contraste a los estudios de observación de tratamientos habituales en los que la adherencia no va mas allá del 30 al 50\%.2-7-10-15-17-18

\section{IV. ¿Es la adherencia al tratamiento farmacológico} predictor del control de la PA del hipertenso? Aunque la frase frecuentemente citada del Dr. Everett Koop, Surgeon General del Gobierno de los EEUU en la década de los 80 , con respecto a la adherencia no necesita un esfuerzo especial de verificación, "drugs don't work in patients who don't take them", que significa "los medicamentos no actúan en quienes no los toman" ${ }^{10}$, la información publicada hasta la fecha ha sido con frecuencia contradictoria o contraintuitiva. Numerosos estudios han mostrado la influencia significativa, aunque generalmente pequeña, de la adherencia medicamentosa en relación al efecto antihi- pertensivo, pero muchos otros no han encontrado una clara relación entre ambas ${ }^{19-20-18-21-15}$. Si bien parte de estas discrepancias pueden deberse a los diferentes métodos utilizados para evaluar la adherencia que van desde encuestas o cuestionarios llenados por los pacientes, a registro de las prescripciones retiradas en las farmacias, conteo de las pastillas residuales en los envases hasta el monitoreo electrónico de la apertura de los envases, método considerado como el más confiable de todos ${ }^{18-22}$, algunos estudios han demostrado una concordancia cercana al $75 \%$ entre este último método y la adherencia evaluada por cuestionarios llenados por los pacientes ${ }^{23-24}$. ¿A qué se deben estas discrepancias entre la adherencia observada y el control de la PA? En la regulación de la PA tanto en individuos normales como en hipertensos hay factores determinantes relacionados con la dieta (contenido de Sodio y Potasio), la actividad física, presencia de obesidad, uso problemático de alcohol, que impactan con mayor intensidad en los hipertensos y que pueden minimizar los efectos del régimen medicamentoso. En Chile un estudio reciente ha comunicado falta de asociación entre PA no controlada y no adherencia (OR 1.01, IC 95\% 0, 78-1,32) con cifras de control de la PA de $56.5 \%$ y adherencia medicamentosa de 37.3 $\%$ en hipertensos seguidos por un año en el Programa de Salud Cardiovascular (PSCV) ${ }^{25}$. Este programa del Ministerio de Salud fue iniciado en el año 2002 en los centros de atención primaria con el objetivo de reducir el riesgo de eventos cardiovasculares en los pacientes mas vulnerables (hipertensos y diabéticos) a través de un acceso sin restricciones a programas multidisciplinarios que además del régimen medicamentoso y seguimiento médico periódico proveen recomendaciones enfocadas en los hábitos y estilos de vida desfavorables ${ }^{14-15}$. Factores asociados a la predicción de falta de adherencia en los hipertensos seguidos en esta cohorte del PSCV incluyeron bajo nivel educacional y de ingresos económicos, sexo masculino, pobre relación médico-paciente, alto nivel de estrés emocional y depresión ${ }^{15-25}$ (Tabla 1). Similares programas multidisciplinarios en EEUU han mostrado también resultados muy por encima del promedio de estudios hechos en ese país en el control del hipertenso ${ }^{26-27}$.

\section{V. ¿Qué métodos pueden favorecer una mejor adheren-} cia medicamentosa antihipertensiva en la práctica habitual? En primer lugar, hay que identificar el problema a través de indicadores importantes: falta de respuesta al tratamiento prescrito, frecuentes fallas en acudir a control, no retiro de las prescripciones en la farmacia. Compartir con el paciente la preocupación e interés de ayudarlo. Explicar 
en cada control la importancia del tratamiento y adaptar éste en lo posible a la realidad y posibilidades del paciente. No caer en la inercia terapéutica y reforzar en cada visita y oportunidad de contacto la importancia de un estilo de vida y hábitos saludables.

En los últimos 10-15 años se ha utilizado la telefonía celular para enviar mensajes recordatorios a los pacientes insistiendo en el uso de sus medicamentos. La gran mayoría de los estudios publicados han informado resultados favorables en mejorar la adherencia a corto plazo (alrededor de 6 meses) incluyendo un estudio recientemente comunicado en Chile ${ }^{28}$. Sin embargo, hasta la fecha no existe suficiente información de resultados favorables en hipertensos por períodos más largos ${ }^{29}$.

\section{Conclusiones}

La adherencia farmacológica es un complejo proceso multifacético en el que están envueltos numerosos actores y que representa solo uno de los factores que determinan el control satisfactorio de la PA. Una mejor adherencia y un control satisfactorio de la PA pueden ser alcanzados en gran medida por un proveedor dispuesto a dedicar suficiente tiempo e interés en el manejo integral del hipertenso. Programas multidisciplinarios enfocados en esta diversidad de componentes han publicado resultados superiores de control de la PA en países con diferentes niveles de desarrollo económico como son Chile y los EEUU.

\begin{tabular}{|c|c|c|c|c|}
\hline & $\begin{array}{r}\text { Adherente } \\
\qquad \begin{array}{r}\mathrm{N}=224 \\
\mathrm{n}(\%)\end{array}\end{array}$ & $\begin{array}{r}\text { No } \\
\text { Adherente } \\
\mathrm{N}=376 \\
\mathrm{n}(\%)\end{array}$ & $\begin{array}{l}\text { Odds Ratio } \\
\text { No ajustado } \\
\text { (IC 95\%) }\end{array}$ & $\begin{array}{r}\text { Odds Ratio } \\
\text { Modelo } 2 \\
\text { Ajustado por } \\
\text { todas las variables } \\
\text { (IC 95\%) }\end{array}$ \\
\hline Edad $\ddagger$ & $56,1 \pm 7,9 a \ddagger$ & $54,4 \pm 8,3$ a & $0,97(0,96-098) \ddagger$ & $0,96(0,94-0,98) \ddagger$ \\
\hline Sexo masculino & $48(21,2)$ & $112(29,7) \ddagger$ & $1,48(1,18-1,87) \ddagger$ & $1,67(1,21-2,28) \ddagger$ \\
\hline Baja educación (<8 años de educación) & $61(26,9)$ & $127(34,0) \dagger$ & $1,29(1,04-1,60) \ddagger$ & $1,75(1,28-2,40) \ddagger$ \\
\hline Bajo ingreso familiar ( $<\$ 45,000$ por persona/mes) & $58(26,0)$ & $157(41,9) \ddagger$ & $2,20(1,76-2,75) \ddagger$ & $1,83(1,38-2,45) \ddagger$ \\
\hline Farmacoterapia antihipertensiva múltiple & $125(55,6)$ & $157(41,7) \ddagger$ & $0,62(0,50-0,76) \ddagger$ & $0,86(0,65-1,13)$ \\
\hline Inadecuada relación médico-paciente & $45(19,9)$ & $119(31,5) \ddagger$ & $1,85(1,41-2,42) \ddagger$ & $1,68(1,22-2,32) \ddagger$ \\
\hline Alto nivel de estrés emocional-depresión & $49(21,9)$ & $118(31,5) \ddagger$ & $1,75(1,38-2,21) \ddagger$ & $1,92(1,36-2,71) \ddagger$ \\
\hline Escaso apoyo social & $71(31,7)$ & $145(38,5)$ & $1,28(1,04-1,57)$ & $1,10(0,83-1,49)$ \\
\hline Diabetes mellitus & $85(37,4)$ & $116(30,7)$ & $0,83(0,68-1,02)$ & $0,82(0,62-1,10)$ \\
\hline Obesidad (IMC $\geq 30$ kg/m2) & $126(56,6)$ & $225(59,7)$ & $0,99(0,81-1,21)$ & $1,03(0,78-1,35)$ \\
\hline Tabaquismo (fumador actual) & $61(27,4)$ & $98(26,1)$ & $0,86(0,67-1,10)$ & $0,80(0,58-1,10)$ \\
\hline Consumo problemático de alcohol & $22(9,9)$ & $59(16,0) \dagger$ & $1,40(1,04-1,89) \ddagger$ & $1,36(0,87-2,13)$ \\
\hline$\ddagger p<0.01$ & & & & \\
\hline
\end{tabular}

Chacón J, et al. Rev Chil Cardiol. 2014; Libro Resúmenes Congreso: 66. 
1. INSTITUTO NACIONAL DE ESTADISTICA. Estadísticas Vitales. Anuario 2012. Santiago de Chile, Septiembre 2012.

.2. KEARNEY PM, WHELTON M, REYNOLDS K, MUNTNER P, WHELTON PK, HE J. Global burden of hypertension: analysis of worldwide data. Lancet. 2005; 365: 217-223.

2. CHOBANIAN AV, BAKRIS GL, BLACK HR, CUSHMAN WC, GREEN LA, IZZO JL Jr, et al. The seventh report of the Joint National Committee on Prevention, Detection, Evaluation, and Treatment of High Blood Pressure: the JNC 7 report. JAMA. 2003; 289: 2560-2572.

3. THE TASK FORCE FOR THE MANAGEMENT OF ARTERIAL HYPERTENSION OF THE EUROPEAN SOCIETY OF HYPERTENSION (ESH) AND OF THE EUROPEAN SOCIETY OF CARDIOLOGY (ESC). 2013 ESH/ESC Guidelines for the management of arterial hypertension. European Heart Journal, 2013; 34: 2159-2219.

4. BLOOM DE, CAFIERO ET, JANÉ-LLOPIS E, ABRAHAMS-GESSEL S, BLOOM LR, FATHIMA S, et al. The global economic burden of non-communicable diseases. Geneva: World Economic Forum, 2011.

5. MINISTERY OF HEALTH (Ministerio de Salud, MINSAL), National Health Survey, Chile. Santiago, 2009-2010; 1.

6. PICKERING TG. Why are we doing so badly with the control of hypertension? Poor compliance is only part of the story. $\mathrm{J}$ Clin Hypertens 2001, 3:179-82.

7. CHOBANIAN AV. The hypertension paradox - more uncontrolled disease despite improved therapy. N Engl J Med 2009; 361:878-88.

8. FRANCO OH, PEETERS A, BONNEAUX L, DE LAET C. Blood pressure in adulthood and life expectancy with cardiovascular diseases in men and women life course analysis. Hypertension 2005; 46: 280-286
9. SABATE E. World Health Organization. Adherence to Long Term Therapies: Evidence for Action. Geneva: World Health Organization; 2003.

10. OSTERBERG L, BLASHKE T. Adherence to Medication. N Engl J Med 2005; 353: 487-97

11. MOZAFFARIAN D1, FAHIMI S, SINGH GM, MICHA R, KHATIBZADEH S, ENGELL RE, et al. Global sodium consumption and death from cardiovascular causes. N Engl J Med 2014; 371: 624-34.

12. PHILLIPS LS, BRANCH WT, COOK CB, DOYLE JP, EL-KEBBII M, GALLINA DL, et al. Clinical inertia. Ann Intern Med 2001; 135: 825-834.

13. DUNBAR-JACOB J, ERLEN JA, SCHLENK EA, RYAN CM, SEREIKA SM, DOSWELL WM. Adherence in chronic disease. Annu Rev Nursing Res 2000; 18: 48-90.

14. SANDOVAL D, BRAVO M, KOCH E, GATICA S, AHLERS I, HENRÍQUEZ O, et al. Overcoming barriers in the management of hypertension. The experience of the Cardiovascular Health Program in Chilean Primary Health Care Centers. Int J Hypertens. 2012; 2012: 405892.

15. SANDOVAL D, CHACÓN J, MUÑOZ R, ENRIQUEZ O, KOCH E, ROMERO T. Influencia de factores psicosociales en la adherencia al tratamiento farmacológico antihipertensivo . Resultados de una cohorte del Programa de Salud Cardiovascular de la Región Metropolitana, Chile. Rev Med Chile 2014; 142: 1245-1252.

16. EZE-NLIAM CM, THOMBS BD, LIMA BB, SMITH CG, ZIEGELSTEIN RC. The association of depression with adherence to antihypertensive medications: a systematic review. J Hypertens 2010; 28:1785-95.

17. MORISKY DE, ANG A, KROUSEL-WOOD M, WARD HJ. Predictive validity of a medication adherence measure in an outpatient setting. J Clin Hypertens 2008; 10: 348-354. 
18. SCHROEDER K, FAHEY T, HAY AD, MONTGOMERY A, PETERS T. Relationship between medication adherence and blood pressure in primary care: a prospective study. J Hum Hypertens, 2006; doi:10.1038/sj.jhh.1002011.

19. BRAMLEY TJ, GERBINO PP, NIGHTENGALE BS, FRECH-TAMAS F. Relationship of blood pressure control to adherence with antihypertensive monotherapy in 13 managed care organizations. J Manag Care Pharm 2006;12:239-45.

20. MAZZAGLIA G, AMBROSIONI E, ALACQUA M, FILIPPI A, SESSA E, IMMORDINO V, et al. Adherence to antihypertensive medications and cardiovascular morbidity among newly diagnosed hypertensive patients. Circulation 2009;120: $1598-1605$.

21. CLAXTON AJ, CRAMER J, PIERCE C. A systematic review of the associations between dose regimens and medication compliance. ClinTher 2001; 23:1296-310.

22. WETZELS GEC, NELEMANS P, SCHOUTEN JS, PRINS MH. Facts and fiction of poor compliance as a cause of inadequate blood pressure control: a systematic review. J Hypertens 2004; 22: 1849-1855.

23. CHOO PW, RAND C, INUI TS, LEE ML, CAIN E, CORDEIRO-BREAULT $\mathrm{M}$, et al. Validation of patient reports, automated pharmacy records, and pill counts with electronic monitoring of adherence to antihypertensive therapy. Medical Care 1999; 37: 846-867.
24. GARBER MC, NAU D, ERICKSON SR, AIKENS JE, LAWRENCE JB. The concordance of self-report with other measures of medication adherence: A summary of the literature. Med Care. 2004; 42: 649-52.

25. CHACON J, SANDOVAL D, MUÑOZ R, ROMERO T. Evaluación del control de la Presión Arterial y adherencia terapeútica en hipertensos seguidos en el Programa de Salud Cardiovascular. Asociación con características clínicas, socioeconómicas y psicosociales. Rev Chil Cardiol . 2014; Libro Resúmenes Congreso: 65.

26. MARGOLIS KL, PILLER LB, FORD CF, HENRIQUEZ MA, CUSHMAN WC, EINHORN PT, et al. Blood pressure control in hispanics in the antihypertensive and lipid-lowering treatment to prevent heart attack trial (ALLHAT). Hypertension 2007; 50: 854-861.

27. JAFFEE MG, LEE GA, YOUNG JD, SIDNEY S, GO AS. Improved blood pressure control associated with a large-scale hypertension program. JAMA. 2013;310:699-705.

28. VARLETA P, AKEL C, ACEVEDO M, SALINAS C, PINO J, GARCÍA A. et al. Impacto de mensajería de texto sobre la adherencia al tratamiento anti hipertensivo. Rev Chil Cardiol . 2014; Libro Resúmenes Congreso: 66.

29. VERVLOET M, LINN AJ, VAN WEERT JCM, DE BAAKER D Y, BOUVY ML. The effectiveness of interventions using electronic reminders to improve adherence to chronic medication: a systematic review of the literature J Am Med Inform Assoc 2012; 19:696-704. 